\title{
Outcomes in cases of lumbar degenerative spondylolisthesis more than 5 years after treatment with minimally invasive decompression: examination of pre- and postoperative slippage, intervertebral disc changes, and clinical results
}

\author{
Gen Mori, MD, Yasuo Mikami, MD, PhD, Yuji Arai, MD, PhD, Takumi Ikeda, MD, PhD, \\ Masateru Nagae, MD, PhD, Hitoshi Tonomura, MD, PhD, Ryota Takatori, MD, PhD, \\ Koshiro Sawada, MD, PhD, Hiroyoshi Fujiwara, MD, PhD, and Toshikazu Kubo, MD, PhD \\ Department of Orthopaedics, Graduate School of Medical Science, Kyoto Prefectural University of Medicine, Kyoto, Japan
}

OBJECT There are reports that fusion is the standard treatment of choice for cases of lumbar degenerative spondylolisthesis (LDS) associated with lumbar spinal canal stenosis with a large degree of slippage. The reasons why, however, have not been clarified. On the other hand, it is known that the progress of slippage decreases and restabilization occurs over the natural course of LDS. Therefore, if minimally invasive decompression could be performed, there would be little possibility of it influencing the natural course of LDS, so it would not be necessary to include preoperative percentage slip in the criteria for the selection of fusion. This study examined the course of LDS cases more than 5 years after treatment with minimally invasive decompression to determine whether pre- and postoperative slippage and disc changes influence the clinical results.

METHODS A total of 51 intervertebral segments in 51 cases with the chief complaint of radicular or cauda equina symptoms due to lumbar spinal canal stenosis were examined after prospective treatment with minimally invasive decompression for LDS. The mean age of the patients at the time of surgery was 66.7 years and the mean follow-up period was 7 years 4 months. Minimally invasive decompression was performed regardless of the degree of low-back pain or percentage slip. The outcome variables were clinical results and changes in imaging findings.

RESULTS Over the follow-up period, postoperative percentage slip increased and disc height decreased, but the Japanese Orthopaedic Association score improved. Regardless of the preoperative percentage slip, disc height, or degree of intervertebral disc degeneration or segmental instability, the clinical results were favorable. In the high preoperative percentage slip group, low disc height group, and progressive disc degeneration group, there was little postoperative progress of slippage. In the group with a postoperative slippage increase of more than $5 \%$, slippage increased significantly at postoperative year 2 , but no significant difference was observed at the final follow-up.

CONCLUSIONS When minimally invasive decompression was performed to treat LDS, the postoperative change in slippage was no different from that during the natural course. Furthermore, regardless of the degree of preoperative slippage or intervertebral disc degeneration, the clinical results were favorable. Also, the higher the preoperative percentage slip and the more that disc degeneration progressed, the more the progress of postoperative slippage decreased. Because the postoperative progress of slippage decreased, it is believed that even after minimally invasive decompression, restabilization occurs as it would during the natural course. If minimally invasive decompression can be performed to treat LDS, it is believed that preoperative percentage slip and intervertebral disc degeneration do not have to be included in the appropriateness criteria for fusion.

http://thejns.org/doi/abs/10.3171/2015.6.SPINE141298

KEY WORDS lumbar degenerative spondylolisthesis; percentage slip; intervertebral disc degeneration; minimally invasive decompression

ABBREVIATIONS DHI = disc height index; JOA = Japanese Orthopaedic Association; LDS = lumbar degenerative spondylolisthesis.

SUBMITTED December 22, 2014. ACCEPTED June 11, 2015.

INCLUDE WHEN CITING Published online November 27, 2015; DOI: 10.3171/2015.6.SPINE141298. 
$\mathrm{D}$ ECOMPRESSION alone, or decompression with fusion, are considered to be the treatments of choice for lumbar degenerative spondylolisthesis (LDS) associated with lumbar spinal canal stenosis. However, there is currently no consensus on the criteria for selecting these two procedures. ${ }^{9,20}$ It has been reported that for cases with a large degree of slippage, the standard procedure is fusion, ${ }^{6}$ but the evidence for this has not been clarified. On the other hand, the progress of slippage decreases during the natural course of LDS, and restabilization occurs. In light of this, it is believed that if minimally invasive decompression is performed, which has little influence on segmental instability and little possibility of influencing the natural course of LDS, there is no need to include the preoperative degree of slippage in the criteria for selecting fusion. Beginning in 2003, LDS cases at our hospital with the chief complaint of radicular or cauda equina symptoms due to lumbar spinal canal stenosis have been treated with decompression using the METRx lumbar endoscopic system (Medtronic Sofamor Danek) regardless of the degree of low-back pain or percentage slip. Because this procedure is minimally invasive and has little impact on facet joints or surrounding soft tissue, even if there is a large degree of preoperative slippage or disc degeneration, it is possible that postoperative restabilization will occur without changing the natural course of LDS. Taking the above into consideration, in this study minimally invasive decompression alone was performed prospectively for LDS cases and the clinical results and postoperative changes in imaging findings were examined to determine the influence of preoperative and postoperative slippage and disc changes on postoperative course.

\section{Methods}

\section{Patient Selection}

The patients were 63 consecutive cases of LDS with the chief complaint of radicular or cauda equina symptoms due to lumbar spinal canal stenosis that did not respond to conservative treatment. Patients were surgically treated by minimally invasive decompression. The procedure was performed regardless of the degree of low-back pain, percentage slip, or stenosis type. Fifty-three patients were examined more than 5 years after surgery. Two cases with slippage at 2 disc levels or more were excluded. Thus, a total of 51 patients were included in this study. The mean age of the patients at the time of surgery was 66.7 years (range 46-85 years) and the mean follow-up duration was 7 years 4 months (range 5 years to 10 years 2 months). All patients had single-level DLS and the mean percentage slip was $15.3 \%$ (range 5.4\%-28.8\%). The Meyerding Grading System classification was Grade I for 47 disc levels and Grade II for 4 disc levels. The demographic characteristics of patients are shown in Table 1. As in a previous study, ${ }^{8}$ the degree of stenosis was evaluated at 4 levels using axial MR images. Nearly all cases had moderate or greater stenosis: 0 were normal, 2 were mild, 6 were moderate, and 26 were severe. In this study, all cases had central and/or lateral stenosis with no associated foraminal stenosis observed. This study was conducted with the approval of the ethics committee of the Kyoto Prefectural University of Medicine.
TABLE 1. Demographic characteristics of the patients in the study

\begin{tabular}{cc}
\hline Variable & Value \\
\hline No. of patients & 51 \\
\hline Males/females & $23 / 28$ \\
\hline Age (yrs) & 66.7 \\
\hline Mean & $46-85$ \\
\hline Range & \\
\hline Follow-up period & \\
\hline Mean & 15.3 \\
\hline Range & mos \\
\hline Percentage slip & 10 yrs 2 mos \\
\hline Mean & $5.4-28.8$ \\
\hline Range & \\
\hline Meyerding Grade & 47 \\
\hline I & 4 \\
\hline II & 0 \\
\hline III & 0 \\
\hline IV &
\end{tabular}

\section{Operative Method}

Microendoscopic decompression using a METRx lumbar endoscopic system (Medtronic Sofamor Danek) was performed according to the method reported by Weiner et al. ${ }^{21}$ Initially, when microendoscopic posterior decompression was performed, a paraspinal approach was used for microendoscopic laminotomy. ${ }^{16,21,22}$ From October 2004, treatment was changed to an improved method developed at our department using a midline approach ${ }^{14}$ providing a bilateral symmetrical field of view. As a result, 11 cases were treated with a paraspinal approach and 40 with a midline approach.

\section{Paraspinal Approach}

In the paraspinal approach, serial dilators are passed to dilate the lumbar paraspinal muscles and to retract the lumbodorsal fascia via a $20-\mathrm{mm}$ midline incision. A $16-$ $\mathrm{mm}$ tubular retractor is then passed over the dilators and the endoscope is attached to the tubular retractor. The inferior edge of the lamina and the medial edge of the facet joint are identified, and a laminotomy plus facetectomy are performed using an air drill and Kerrison rongeur. The anterior portion of the lamina on the contralateral side is then removed. The dura mater and the nerve root are exposed after resection of the ligamentum flavum. ${ }^{16,21,22}$

\section{Midline Approach}

In the midline approach, a $20-\mathrm{mm}$ incision is made in the skin in the midline. The spinous process on the cranial side is partially excised, and incisions along the ligament fiber are made in the midline of the supraspinous and interspinous ligaments to expose the ligamentum flavum. After the lamina and the inferior parts of the bilateral facet joints are adequately excised, a 16-mm tubular retractor is inserted and an endoscope is attached. The cranial third of the lower adjacent lamina is then removed to free 


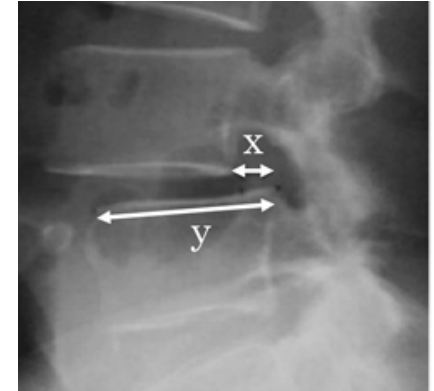

percentage $\operatorname{slip}=\mathrm{x} / \mathrm{y} \times 100$

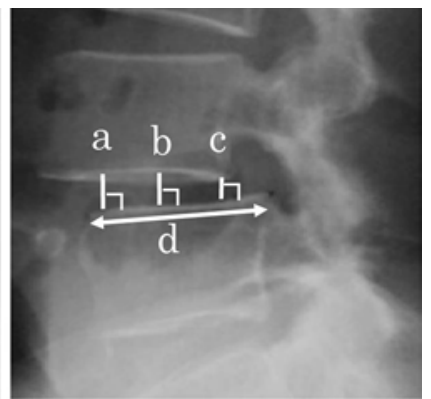

$\mathrm{DHI}=(\mathrm{a}+\mathrm{b}+\mathrm{c}) / \mathrm{d}$
FIG. 1. Lateral lumbar radiographs showing the calculation of the percentage slip and DHI. $a=$ anterior disc height; $b=$ central disc height; $c$ $=$ posterior disc height; $d=$ anteroposterior width of the lower vertebral body; $x$ = slippage width; $y=$ anteroposterior width of the lower vertebral body.

the caudal margin of the ligamentum flavum. After partial laminotomy of the caudal half of the upper adjacent lamina, a domelike expansion is performed by removing the inner laminar plate to free the cranial margin of the ligamentum flavum. The bilateral facet joint is then undercut to expose the lateral margin of the ligamentum flavum, exposing the bilateral nerve roots. ${ }^{14}$

\section{Outcome Variables}

Clinical results were determined using Japanese Orthopaedic Association (JOA) Assessment of Treatment for Low Back Pain scores (29-point scale) $)^{5,14}$ and their improvement rate was examined. The number of reoperated cases and their causes were also examined. The percentage slip before surgery and at the final follow-up was determined by evaluating the degree of slippage observed on lateral lumbar radiographs, and the disc height index (DHI) was determined by evaluating disc height. The percentage slip was calculated as the amount of slippage divided by the anteroposterior width of the lower vertebra. DHI was calculated as the sum of the anterior, central, and posterior disc heights divided by the anteroposterior width of the lower vertebral body (Fig. 1). Depending on the degree of preoperative slippage, patients were classified into the 4 groups of low $(<10 \%)$, mild $(10 \%-15 \%)$, moderate $(15 \%-20 \%)$, and severe $(>20 \%)$ slippage. Preoperative percentage slip was compared with preoperative DHI, the postoperative degree of progression of slippage (the value calculated by subtracting the preoperative percentage slip from the percentage slip at the final follow-up examina- tion), and the JOA score improvement rate. Depending on the preoperative DHI, patients were classified into the 3 groups of high (>0.6), moderate (0.4-0.6), and low DHI $(<0.4)$. Preoperative DHI was compared with the postoperative degree of slippage progression and JOA score improvement rate. Depending on the postoperative increase in percentage slip, patients were classified into 2 groups ( $<5 \%$ increase and $>5 \%$ increase), and the relationship to JOA scores was examined. For patients in the group with a postoperative percentage slip increase of $>5 \%$, the percentage slip was evaluated chronologically.

To determine segmental dynamic instability, lateral lumbar radiographs were used to measure pre- and postoperative segmental range of motion, kyphotic angle during forward flexion, and sagittal translation. Instability was defined as a segmental range of motion of $\geq 10^{\circ}$, $\mathrm{ky}$ photic angle during forward flexion of $\geq 10^{\circ}$, or sagittal translation of $\geq 5 \mathrm{~mm}$. The relationship between the presence or absence of pre- and postoperative instability and JOA score was examined.

Signal intensity changes in MR images of vertebral endplates and discs were evaluated using the Modic classification (normal, Type I, Type II, and Type III) ${ }^{16}$ and Schneiderman's classification ${ }^{19}$ (normal, intermediate, marked, and absent), respectively (Fig. 2). The relationship between these classifications and the degree of progress of postoperative slippage and JOA score improvement rate was examined.

\section{Statistical Analysis}

The multiple comparison test and paired t-test were used for statistical analysis.

\section{Results}

The JOA score improved significantly from a mean preoperative score of 14.3 to a mean postoperative score of 23.6 points $(\mathrm{p}<0.01)$. There were 7 reoperated cases (13.7\%): 2 with stenosis and hernia at another level (reoperation at 20 and 61 months, respectively), 2 with restenosis (reoperation at 45 and 94 months, respectively), and 3 with progressive degenerative scoliosis (reoperation at 22, 25 , and 84 months, respectively). Additional surgery was performed with fusion for 2 of them. None of these 7 cases had a preoperative percentage slip of more than $20 \%$.

The mean percentage slip increased significantly from $14.6 \%$ preoperatively to $17.5 \%$ postoperatively $(\mathrm{p}<0.01)$, and DHI decreased significantly from 0.54 preoperatively

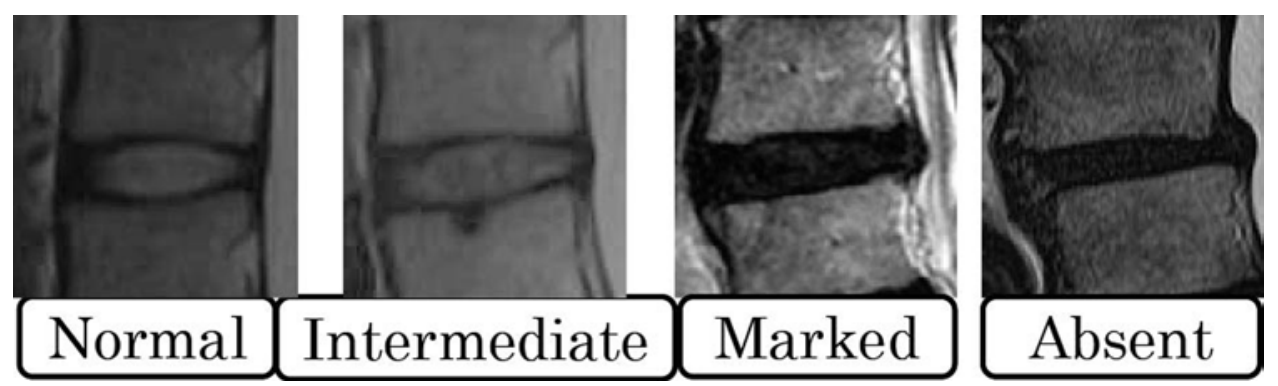

FIG. 2. Schneiderman's classification into 4 groups using T2-weighted MR images to determine the degree of intervertebral disc degeneration. 


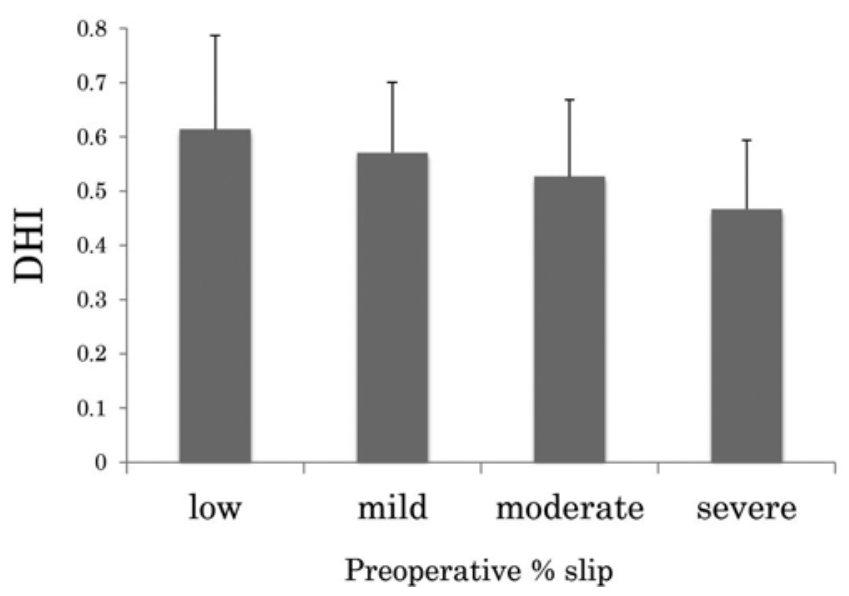

FIG. 3. Graph of DHI according to preoperative percentage slip.

to 0.44 postoperatively $(\mathrm{p}<0.01$ ). Preoperative percentage slip was classified as low slippage at 11 disc levels, mild slippage at 19 disc levels, moderate slippage at 10 disc levels, and severe slippage at 10 disc levels. Postoperative percentage slip was classified as low slippage at 6 disc levels, mild slippage at 12 disc levels, moderate slippage at 14 disc levels, and severe slippage at 18 disc levels, which showed that slippage had progressed compared with preoperative values. Preoperative DHI was classified as high disc height at 19 disc levels, moderate disc height at 19 disc levels, and low disc height at 11 disc levels. Postoperative DHI was classified as high disc height at 10 disc levels, moderate disc height at 19 disc levels, and low disc height at 20 disc levels, showing a decrease compared with preoperative values.

It was observed that the higher the preoperative percentage slip, the lower the preoperative DHI (Fig. 3). Furthermore, the postoperative progress of slippage was less in cases with a higher preoperative percentage slip (Fig. 4 left). No significant difference was observed in JOA score improvement rates by preoperative percentage slip (Fig. 4 right). Postoperative slippage progressed less in the group with low preoperative DHI compared with the group with high DHI (Fig. 5 left). No significant difference was ob-

(\%)

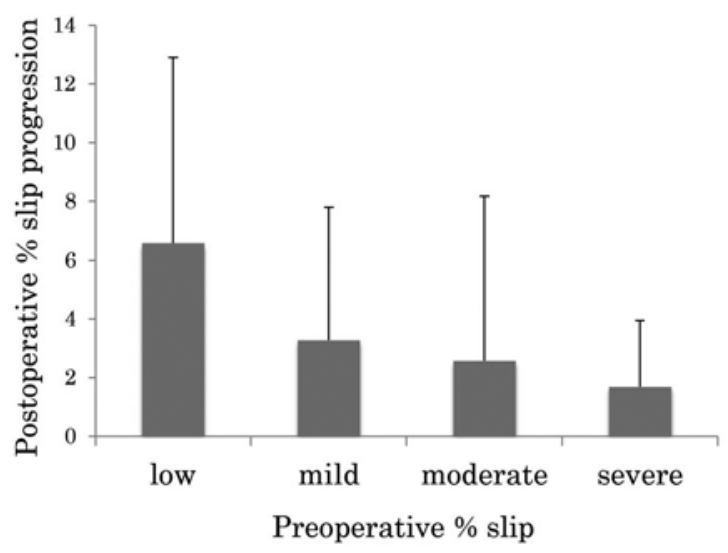

served in JOA score improvement rates by preoperative DHI (Fig. 5 right). There were 16 cases $(32.7 \%)$ with a postoperative percentage slip increase of $>5 \%$ and 33 cases $(67.3 \%)$ with a postoperative percentage slip increase $<5 \%$. The JOA score improvement rate was significantly higher $(\mathrm{p}=0.03)$ in the group with a postoperative percentage slip increase $>5 \%$ compared with the group with a postoperative percentage slip increase $<5 \%$ (Fig. 6). The percentage slip in the group with a postoperative percentage slip increase of $>5 \%$ increased significantly from the preoperative value of $13.6 \%$ to the postoperative year 2 value of $19.9 \%$, but no significant difference was observed at the final follow-up (Fig. 7). The severity of stenosis had no influence on JOA score improvement rates and postoperative slippage progression.

The mean JOA improvement rate for the 6 cases with preoperative segmental instability was $57.3 \%$. In 5 of these cases, instability disappeared after surgery. The mean JOA score improvement rate for the 7 cases with postoperative segmental instability was $58.7 \%$. In 6 of these cases there was no preoperative instability. No significant difference was observed between pre- and postoperative segmental instability and mean JOA score improvement rate.

Preoperative Modic classifications were normal at 30, Type I at 3, Type II at 2, and Type III at 2 disc levels, with normal being the most common classification. However, the postoperative classifications were normal at 19, Type I at 5, Type II at 9, and Type III at 9 disc levels, with fewer disc levels classified as normal and an increase in those classified as Type II or Type III. No relationship was observed between the degree of vertebral endplate degeneration and the degree of postoperative slippage or JOA score improvement rate. Preoperative Schneiderman's classifications were intermediate at 7 , marked at 22 , and absent at 8 disc levels, with no normal cases observed. The postoperative classifications were intermediate at 2 , marked at 17 , and absent at 18 disc levels, showing an increase in the number of disc levels classified as absent. In the group with preoperative progressing disc degeneration, there was little postoperative increase of slippage and no significant difference was observed between JOA score improvement rates (Fig. 8).

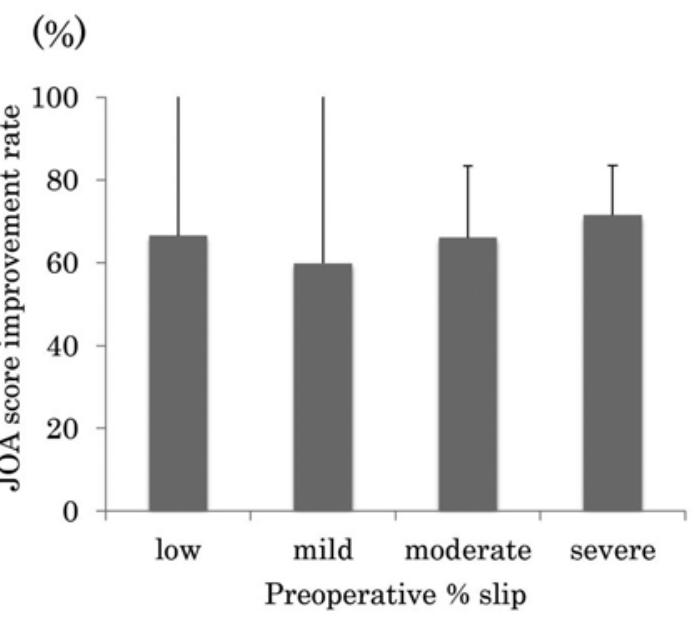

FIG. 4. Graphs of postoperative percentage slip progression by preoperative percentage slip (left) and JOA score improvement rate by preoperative percentage slip (right). 
(\%)

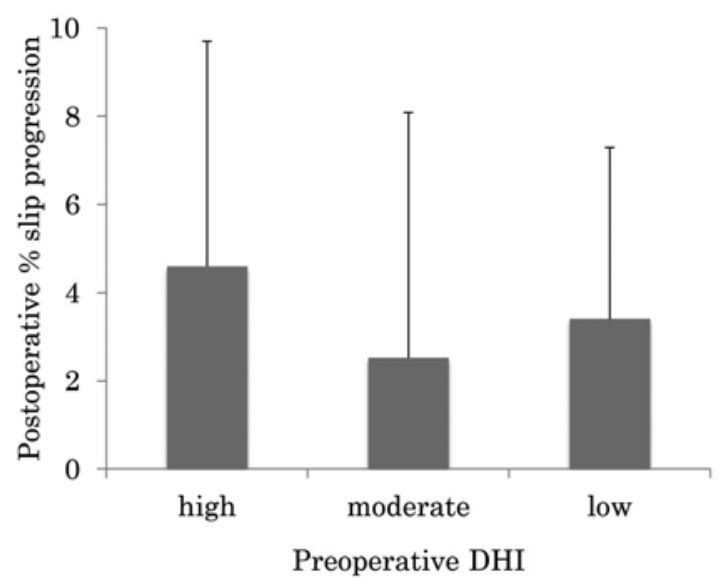

(\%)

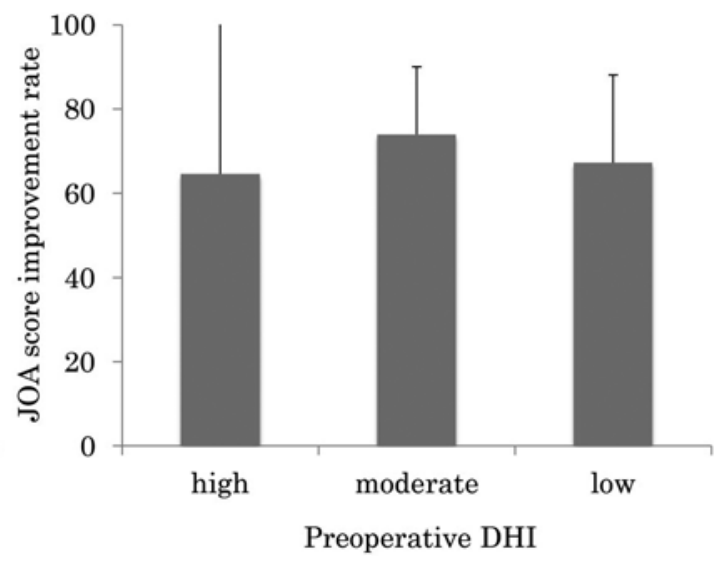

FIG. 5. Graphs of postoperative percentage slip progression by preoperative DHI (left) and JOA score improvement rate by preoperative $\mathrm{DHI}$ (right).

\section{Illustrative Case}

A 73-year-old man underwent microendoscopic decompression via a midline approach for LDS at L5-S1. Preoperative percentage slip, DHI, and JOA score were $24.6 \%, 0.33$, and 15 , respectively. The percentage slip, DHI, and JOA score at the final follow-up (7 years and 0 months postoperatively) were $26.2 \%, 0.30$, and 26 , respectively. There was a high degree of preoperative slippage, but it had not progressed at the final follow-up, and the JOA score improvement rate was $78.6 \%$ (Fig. 9).

\section{Discussion}

The surgical treatments for LDS are considered to be

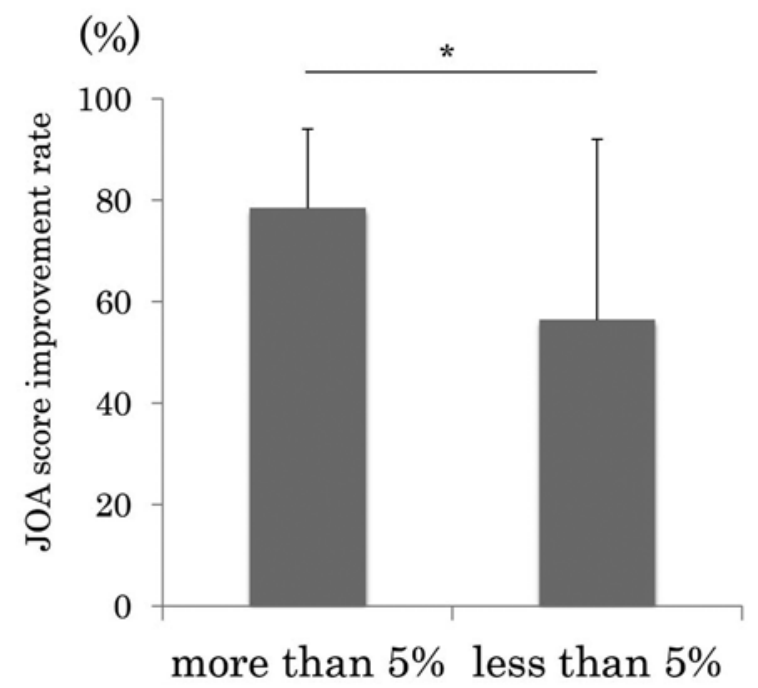

The group with increased postoperative $\%$ slip

$$
\text { * } \mathrm{P}<0.05
$$

FIG. 6. Graph of JOA score improvement rate by postoperative increase in percentage slip. The improvement in JOA score in the group with postoperative slippage that had progressed by $>5 \%$ was significantly superior to that in the group with slippage that had progressed by $<5 \%$. decompression alone or decompression with fusion, but there are wide-ranging opinions concerning which procedure to select. ${ }^{3,9,20}$ There have been reports that segmental instability in LDS is related to the cause and progress of slippage. ${ }^{1,4,7}$ For this reason, because conventional procedures for decompression involving surgical invasion of facet joints and surrounding soft tissue could increase instability and exacerbate clinical symptoms, decompression combined with fusion is recommended. Based on randomized controlled trials, Martin et al. ${ }^{10}$ recommend fusion using instrumentation while Resnick et al. ${ }^{18}$ recommend fusion as a guideline. It has also been reported, however, that there is no clear relationship between segmental instability and clinical symptoms. ${ }^{12,13}$ Furthermore, in recent years, noninvasive procedures have been developed and introduced that enable decompression with minimal influence on segmental stability. ${ }^{14,17}$ Matsudaira et al., ${ }^{11}$ Försth et al., ${ }^{2}$ and Minamide et al. ${ }^{15}$ have reported obtaining favorable results with decompression alone. We have also performed microendoscopic decompression for all cases of LDS with the chief complaint of radicular or cauda equina symptoms due to lumbar spinal canal stenosis, regardless of the presence or absence of low-back pain, the degree of slippage, instability, or stenosis type, and obtained favorable clinical results. The invasiveness to the facet joint using our method was believed to be responsible for these good results. This method has been useful for treating LDS.

Matsunaga et al. ${ }^{13}$ examined the natural course of degenerative spondylolisthesis and reported that at follow-up after more than 5 years and after more than 10 years, the percentage slip had increased by more than 5\% in 30\% (12 of 40) and $34 \%$ (49 of 145) 12 of cases, respectively. In our study, at follow-up after more than 5 years (mean 7 years 4 months), the percentage slip had increased by more than $5 \%$ in $30 \%$ of cases. Consequently, it is believed that microendoscopic decompression does not change the natural course of LDS. In addition, although there was a significant increase in the mean percentage slip from $14.9 \%$ preoperatively to $16.9 \%$ postoperatively, the postoperative JOA score showed a significant improvement compared 

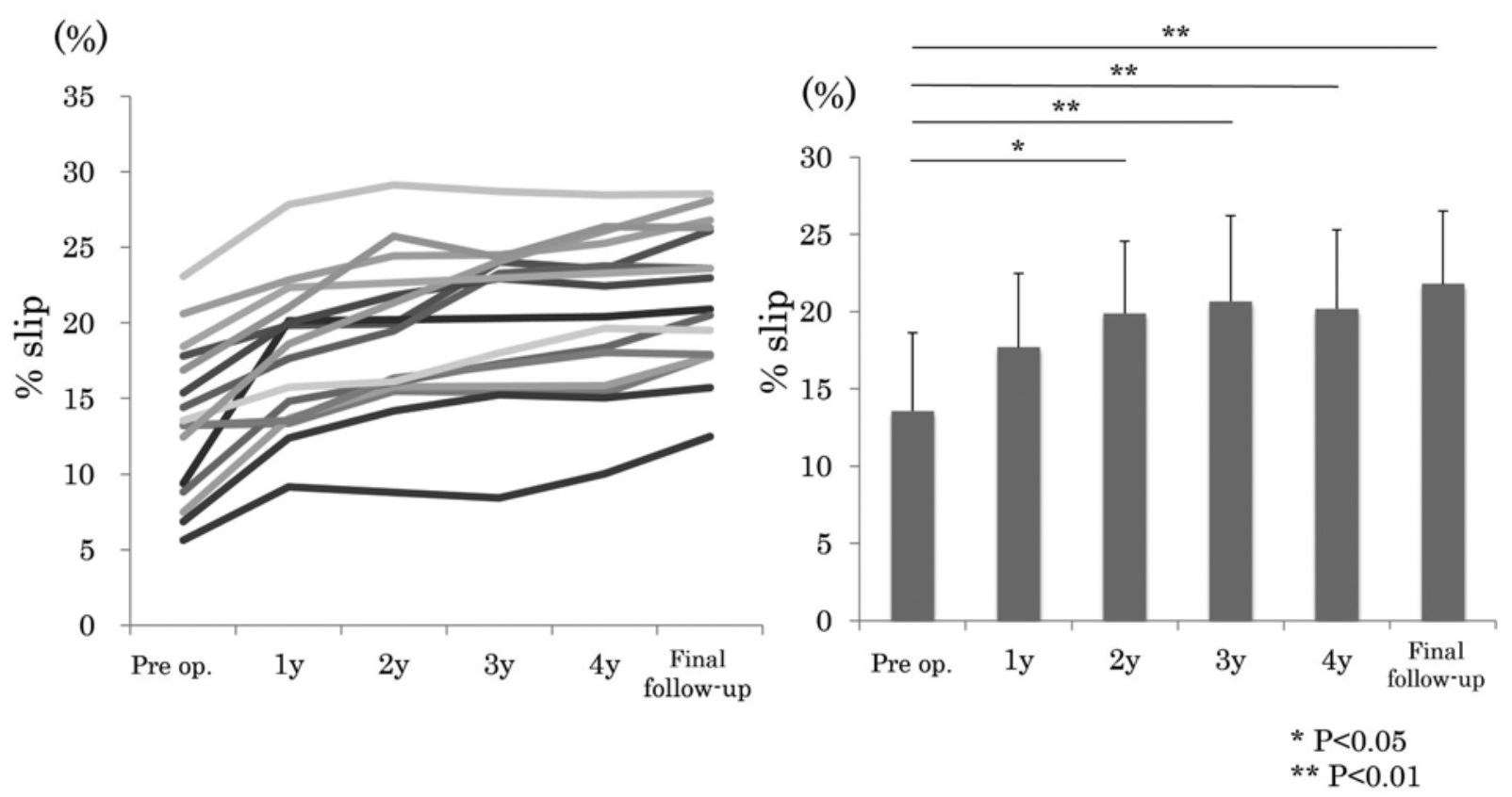

FIG. 7. Change in percentage slip over time in cases with a postoperative percentage slip increase of $>5 \%$. Individual change (left) as well as average time-dependent change (right) is shown.

with the preoperative score, and the clinical results were favorable. With the microendoscopic decompression procedure we used, because posterior decompression improved neurological symptoms, minimized invasive surgery involving paravertebral muscle, and enabled preservation of facet joints and posterior supporting tissue, it was believed that it had minimal influence on the natural course of slippage. It was also believed that there was no clear relationship between the progress of postoperative slippage and clinical results.

A 10 -year follow-up study of LDS by Matsunaga et al. examined the results obtained with conservative treatment. Disc height decreased in 85 of 94 cases, but because there was remission of low-back pain during the course, ${ }^{12}$ it is possible that as lumbar degeneration progresses, restabili- zation occurs between vertebral bodies, ${ }^{6}$ thereby improving low-back pain. In our study, in the group with a high preoperative percentage slip, low disc height, and progressing disc degeneration, postoperative progression of slippage was minimal and clinical results were favorable regardless of preoperative slippage, disc degeneration, or the postoperative degree of progression of slippage. In addition, in the group with a postoperative slippage increase of $>5 \%$, even though there was a significant increase in the percentage slip at postoperative year 2, thereafter and up to the final follow-up there was no change. These results point to the possibility that if minimally invasive decompression is used, the progress of postoperative slippage is reduced and restabilization occurs in the same way as during the natural course of LDS. In this study, compared
(\%)

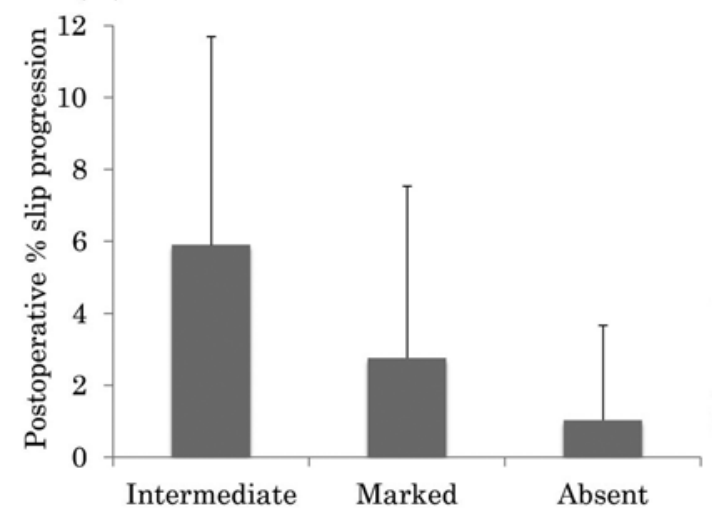

Preoperative Schneiderman's classification
(\%)

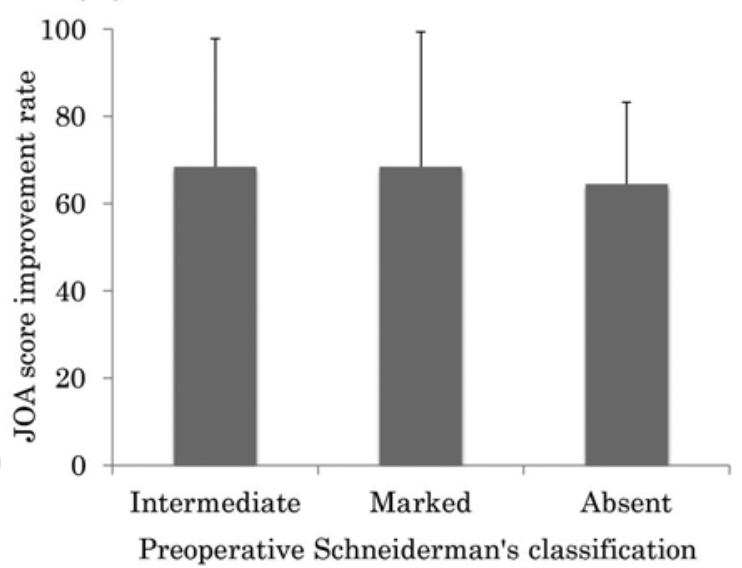

FIG. 8. Graphs of postoperative percentage slip progression (left) and JOA score improvement rate (right) according to preoperative Schneiderman's classification. 


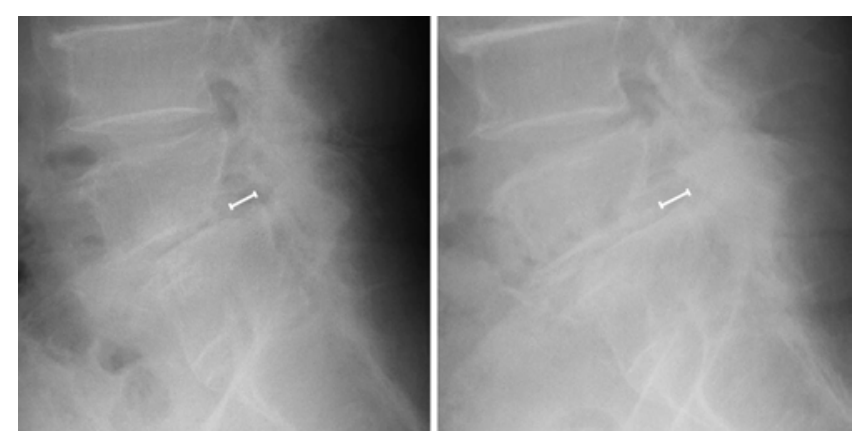

FIG. 9. Preoperative (left) and final follow-up (postoperative year 7; right) lateral lumbar radiographs of a representative case. White line represents slippage width.

with the group with postoperative percentage slip progressing by $<5 \%$, the clinical results were more favorable in the group with postoperative percentage slip progressing by $\geq 5 \%$. It was believed that this could have been due to the large number of cases with segmental stability in the group with high progression of percentage slip. Taking the above into consideration, it is believed that if minimally invasive decompression is performed, there is no need to include preoperative percentage slip and preoperative disc degeneration in the appropriateness criteria for fusion.

The use of minimally invasive decompression has its limitations, however, and it cannot be used for all cases of LDS. In this study there were 7 cases that underwent reoperation, 2 of which required fusion, but there were no cases of reoperation due to slippage progression. Also, there were no cases requiring reoperation due to the progression of postoperative instability. Of the 2 cases that required fusion, 1 case had associated degenerative lumbar scoliosis and the other had foraminal stenosis due to progressive scoliosis. For cases of LDS with associated degenerative scoliosis, it is necessary to examine whether minimally invasive decompression should be combined with fusion.

There were two limitations in this study. First, two surgical approaches for microendoscopic laminotomy were used in the study population and the difference between a paraspinal approach and a midline approach could have influenced the results. Second, this was an uncontrolled study so no comparison could be made between the therapeutic effect of this method and conservative treatment and/or conventional laminectomy, which would represent a relevant control group.

\section{Conclusions}

Favorable clinical results were obtained using minimally invasive decompression to treat LDS regardless of the degree of preoperative slippage or intervertebral disc degeneration. Even in cases with a high preoperative percentage slip and advanced disc degeneration, because slippage had ceased to progress at postoperative year 2 , it was believed that restabilization had occurred as it does during the natural course of LDS. If it is possible to perform minimally invasive decompression to treat LDS, it is believed that it is not necessary to include preoperative percentage slip and intervertebral disc degeneration in the appropriateness criteria for fusion.

\section{References}

1. Cinotti G, Postacchini F, Fassari F, Urso S: Predisposing factors in degenerative spondylolisthesis. A radiographic and CT study. Int Orthop 21:337-342, 1997

2. Försth P, Michaëlsson K, Sandén B: Does fusion improve the outcome after decompressive surgery for lumbar spinal stenosis?: A two-year follow-up study involving 5390 patients. Bone Joint J 95-B:960-965, 2013

3. Herkowitz HN, Kurz LT: Degenerative lumbar spondylolisthesis with spinal stenosis. A prospective study comparing decompression with decompression and intertransverse process arthrodesis. J Bone Joint Surg Am 73:802-808, 1991

4. Iguchi T, Nishida K, Ozaki T, Kitagawa A, Tsumura N, Kakutani K, et al: Grade three disc degeneration is a critical stage for anterior spondylolisthesis in lumbar spine. Eur Spine J 21:2134-2139, 2012

5. Izumida S, Inoue S: [Assessment of treatment for low back pain.] J Jpn Orthop Assoc 60:909-911, 1986 (Jpn)

6. Kirkaldy-Willis WH, Farfan HF: Instability of the lumbar spine. Clin Orthop Relat Res (165):110-123, 1982

7. Knutsson F: The instability associated with disk degeneration in the lumbar spine. Acta Radiol 25:593-609, 1944

8. Lurie JD, Tosteson AN, Tosteson TD, Carragee E, Carrino JA, Kaiser J, et al: Reliability of readings of magnetic resonance imaging features of lumbar spinal stenosis. Spine (Phila Pa 1976) 33:1605-1610, 2008 [Erratum in Spine (Phila Pa 1976) 33:2482, 2008]

9. Mannion AF, Pittet V, Steiger F, Vader JP, Becker HJ, Porchet F: Development of appropriateness criteria for the surgical treatment of symptomatic lumbar degenerative spondylolisthesis (LDS). Eur Spine J 23:1903-1917, 2014

10. Martin CR, Gruszczynski AT, Braunsfurth HA, Fallatah SM, O'Neil J, Wai EK: The surgical management of degenerative lumbar spondylolisthesis: a systematic review. Spine (Phila Pa 1976) 32:1791-1798, 2007

11. Matsudaira K, Yamazaki T, Seichi A, Takeshita K, Hoshi K, Kishimoto J, et al: Spinal stenosis in grade I degenerative lumbar spondylolisthesis: a comparative study of outcomes following laminoplasty and laminectomy with instrumented spinal fusion. J Orthop Sci 10:270-276, 2005

12. Matsunaga S, Ijiri K, Hayashi K: Nonsurgically managed patients with degenerative spondylolisthesis: a 10- to 18 -year follow-up study. J Neurosurg 93 (2 Suppl):194-198, 2000

13. Matsunaga S, Sakou T, Morizono Y, Masuda A, Demirtas AM: Natural history of degenerative spondylolisthesis. Pathogenesis and natural course of the slippage. Spine (Phila Pa 1976) 15:1204-1210, 1990

14. Mikami Y, Nagae M, Ikeda T, Tonomura H, Fujiwara H, Kubo T: Tubular surgery with the assistance of endoscopic surgery via midline approach for lumbar spinal canal stenosis: a technical note. Eur Spine J 22:2105-2112, 2013

15. Minamide A, Yoshida M, Yamada H, Nakagawa Y, Kawai M, Maio K, et al: Endoscope-assisted spinal decompression surgery for lumbar spinal stenosis. J Neurosurg Spine 19:664-671, 2013

16. Modic MT, Steinberg PM, Ross JS, Masaryk TJ, Carter JR: Degenerative disk disease: assessment of changes in vertebral body marrow with MR imaging. Radiology 166:193-199, 1988

17. Pao JL, Chen WC, Chen PQ: Clinical outcomes of microendoscopic decompressive laminotomy for degenerative lumbar spinal stenosis. Eur Spine J 18:672-678, 2009

18. Resnick DK, Watters WC III, Sharan A, Mummaneni PV, Dailey AT, Wang JC, et al: Guideline update for the performance of fusion procedures for degenerative disease of the lumbar spine. Part 9: lumbar fusion for stenosis with spondylolisthesis. J Neurosurg Spine 21:54-61, 2014

19. Schneiderman G, Flannigan B, Kingston S, Thomas J, Dillin WH, Watkins RG: Magnetic resonance imaging in the 
diagnosis of disc degeneration: correlation with discography. Spine (Phila Pa 1976) 12:276-281, 1987

20. Steiger F, Becker HJ, Standaert CJ, Balague F, Vader JP, Porchet F, et al: Surgery in lumbar degenerative spondylolisthesis: indications, outcomes and complications. A systematic review. Eur Spine J 23:945-973, 2014

21. Weiner BK, Walker M, Brower RS, McCulloch JA: Microdecompression for lumbar spinal canal stenosis. Spine (Phila Pa 1976) 24:2268-2272, 1999

22. Yoshida M, Ueyoshi A, Maio K, Kawai M, Nakagawa Y: Surgical procedures and clinical results of endoscopic decompression for lumbar canal stenosis, in Dazawa A, Chen PQ, Chung JY (eds): State of the Art for Minimally Invasive Spine Surgery. Tokyo: Springer-Verlag, 2005, pp 15-24

\section{Disclosures}

The authors report no conflict of interest concerning the materials or methods used in this study or the findings specified in this paper.

\section{Author Contributions}

Conception and design: Mori, Mikmai, Nagae. Acquisition of data: Mori, Mikmai, Nagae. Analysis and interpretation of data: Arai, Mori, Mikmai, Nagae. Drafting the article: Arai, Mori, Mikmai. Critically revising the article: Arai, Mori, Mikmai, Ikeda, Nagae. Reviewed submitted version of manuscript: Arai, Mori, Mikmai, Ikeda, Nagae, Tonomura, Takatori. Approved the final version of the manuscript on behalf of all authors: Arai. Statistical analysis: Mikmai, Takatori. Administrative/technical/material support: Arai, Mikmai, Tonomura, Takatori, Sawada, Kubo. Study supervision: Arai, Mikmai, Ikeda, Nagae, Tonomura, Takatori, Sawada, Fujiwara, Kubo.

\section{Correspondence}

Yuji Arai, Department of Orthopaedics, Graduate School of Medical Science, Kyoto Prefectural University of Medicine, 465 Kajiicho, Kawaramachi-Hirokoji, Kamigyo-ku, Kyoto 602-8566, Japan.email: yarai89046@nike.eonet.ne.jp. 